УДК 616.3009.7-07-08]-053.2

DOI: $10.26435 /$ UC.V0I4(33).330

\author{
А.В. Налетов ${ }^{1}$, Д.А Карпенко르, Л.Ф. Чалая ${ }^{1}$ \\ ${ }^{1} Г 00$ ВПО «Донецкий национальный медицинский университет имени М. Горького», Донецк \\ ${ }^{2}$ КУ «Центр первичной медико-санитарной помощи № 7 г. Донецка», Донецк
}

\title{
ДИФФЕРЕНЦИАЛЬНАЯ ДИАГНОСТИКА ФУНКЦИОНАЛЬНЫХ АБДОМИНАЛЬНЫХ БОЛЕВЫХ РАССТРОЙСТВ У ДЕТЕЙ В CВETE РИМСКОГО КОНСЕНСУСА IV
}

\begin{abstract}
Функциональные гастроинтестинальные расстройства (ФГИР) занимают одно из ведущих мест в структуре патологии органов пищеварения у детей. Одной из наиболее частых причин обращения за медицинской помощью пациентов различного возраста являются жалобы, связанные с наличием абдоминальной боли. На сегодня основополагающим документом, регламентирующим диагностику и лечение ФГИР среди пациентов разного возраста, являются Римские критерии IV (2016) [1]. Функциональные абдоминальные болевые расстройства (ФАБР) в педиатрической практике в данном документе представлены в разделе «Н2» и рассматриваются в возрастной группе пациентов - «дети и подростки». К ФАБР, согласно Римскому консенсусу IV, относят «функциональную диспепсию» (ФД) (Н2a), «синдром раздраженного кишечника» (CPK) (H2b), «абдоминальную мигрень» (H2c), «функциональную неспецифическую абдоминальную боль» (ФНАБ) (H2d). Несмотря на широкую распространенность ФАБР, на сегодня отсутствует четкость понимания патогенетических механизмов и диагностических критериев данных заболеваний среди педиатров и детских гастроэнтерологов. Кроме того, в Международной классификации болезней Х пересмотра среди представленных нозологий, код имеет лишь СРК. В связи с этим наличие у ребенка ФГИР ошибочно маскируется педиатрами под такими диагнозами, как «хронический гастрит», «хронический колит», «панкреатит», «дискинезия желчевыводящих путей» и т.д., с дальнейшим назначением необоснованного медикаментозного лечения. Среди встречающихся в практике диагнозов достаточно часто можно встретить «СРК», однако в большинстве случаев, он ошибочно выставляется при наличии у ребенка лишь изменений характера стула и в ряде случаев даже может не сопровождаться абдоминальным болевым синдромом, что не соот-
\end{abstract}

ветствует диагностическим критериям данного ФГИР; иногда используется диагноз - «ФД», под который суммируют в ряде случаев любую абдоминальную боль в сочетании с диспептическими симптомами. В свою очередь, случаи диагностики «ФНАБ» или «абдоминальной мигрени» остаются единичными, что вовсе не указывает на редкость данных патологий. Эти диагностические ошибки приводят к назначению ребенку необоснованной терапии, и, как следствие, к полипрагмазии и пролонгации сроков лечения и естественному снижению ее эффективности. Начавшись в раннем или дошкольном возрасте, ФГИР при неправильном лечении и продолжающемся воздействии триггерных факторов имеет тенденцию к трансформации клинической симптоматики. Длительное рецидивирующее течение ФГИР, неэффективное лечение приводят к значительному снижению качества жизни, длительной ограниченности в приеме ряда продуктов питания и напитков, изменению туалетных привычек, что обязательно сказывается на психологическом состоянии больного, усугубляя течение заболевания, разочаровывая пациента в отношении к лечению, снижая его комплаентность, а в глобальном смысле приводит к существенному экономическому ущербу для системы здравоохранения за счет прямых и косвенных расходов на лечение таких больных [2-4].

Классификация и диагностика ФГИР основана в первую очередь на оценке симптомов, данных анамнеза, объективного обследования и результатах минимального набора лабораторных исследований, что позволяет в короткие сроки поставить первичный диагноз. Проведение углубленного лабораторного либо инструментального обследования рекомендовано лишь при подозрении на наличии органической па-

(c) А.В. Налетов, Д.А Карпенко, Л.Ф. Чалая, 2019 (c) Университетская Клиника, 2019 
тологии у пациента. Эксперты педиатрического комитета Римских критериев IV приводят перечень потенциальных симптомов тревоги у детей с хронической абдоминальной болью (относятся ко всем ФАБР), наличие которых позволяет исключить у пациента функциональный генез заболевания и направить поиски на наличие органической патологии:

• отягощенный семейный анамнез по наличию воспалительных заболеваний кишечника, целиакии или язвенной болезни;

- персистирующая абдоминальная боль в правом верхнем или правом нижнем квадрантах живота;

- дисфагия, одинофагия (боль при глотании);

- персистирующая рвота;

- гастроинтестинальные потери крови;

- ночная диарея;

- артрит;

- периректальные заболевания;

- необъяснимая потеря массы тела;

- замедление роста и темпов полового развития;

- необъяснимая лихорадка.

\section{Функциональная диспепсия}

Согласно проведенных в США опросов матерей, у 1,4\% детей отмечается боль или дискомфорт в верхней части живота, по крайней мере, 1 раз в неделю [5]; 5-10\% подростков отмечают наличие диспепсических симптомов [6].

ФД - гетерогенное расстройство, связанное с различными патофизиологическими нарушениями. Гипотезы патогенеза ФД включают нарушения моторики желудка, висцеральную гиперчувствительность вследствие центральной или периферической сенсибилизации, минимальное воспаление и генетическую предрасположенность. Установлено нарушение желудочной аккомодации в виде снижения способности желудка к расслаблению в ответ на поступление пищи, снижение порога чувствительности к растяжению. Выявлено, что ФД развивается у $24 \%$ детей после острого бактериального гастроэнтерита. Имеются сведения о роли аллергии к белкам коровьего молока в патогенезе ФД [7-8].

Диагностические критерии ФД должны включать 1 или более из следующих симптомов, продолжительностью по крайней мере 4 дня в месяц:

- чувство переполнения после еды;

- раннее насыщение;

- эпигастральная боль или жжение, не ассоциированные с дефекацией;

- после соответствующей оценки симптомы не могут быть полностью объяснены другим состоянием.
В ФД в настоящее время приняты следующие подтипы:

- Постпрандиальный дистресс-синдром включает беспокоящее чувство переполнения или раннего насыщения после обычного приема пищи. Подтверждают картину вздутие в верхней части живота, постпрандиальная тошнота или чрезмерная отрыжка.

- Эпигастральный болевой синдром включает все следующие симптомы: беспокоящая (достаточно сильная, чтобы мешать нормальной деятельности) локализованная боль или жжение в эпигастральной области. Боль негенерализованная или локализованная в других областях живота, или грудной клетки, не уменьшается после дефекации или отхождения газов. Подтверждающие критерии могут включать (а) боль жгучего характера, но без ретростернального компонента и (b) боль, которая обычно вызывается или уменьшается после приема пищи, но может проявиться во время голода [9].

Соответствие критериям должно наблюдаться в течение не менее 2-х последних месяцев перед диагностикой.

Выполнять эндоскопическое исследование у детей с ФД рекомендовано в следующих ситуациях:

- отягощенный семейный анамнез по язвенной болезни или инфекции H. pylori,

- возраст старше 10 лет,

- сохранение симптомов дольше 6 месяцев,

- симптомы являются достаточно выраженными и влияют на деятельность в повседневной жизни, включая сон [10].

\section{Синдром раздраженного кишечника}

Частота встречаемости СРК у детей разных стран находится в диапазоне 1,2-5,4\% [11-12].

СРК рассматривается как мультифакториальная патология с комплексом патофизиологических факторов. Триггерами СРК могут выступать перенесенный гастроэнтерит, пищевая непереносимость, хронический стресс, отрицательные эмоции или хирургическое вмешательство. Некоторые дети с СРК имеют ректальную гипералгезию. Висцеральная гиперчувствительность может быть связана с психологическими расстройствами (тревога, депрессия, импульсивность, гнев) [13]. Результирующие патофизиологические механизмы вариабельны и индивидуальны. Они включают в себя нарушения моторики, висцеральную гиперчувствительность, повышенную кишечную проницаемость, иммунную активацию, нарушения состава микробиоты, расстройство функционирования оси «мозг-кишечника». Остается не до конца ясным, являются ли обнаруженные изменения состава 
кишечной микробиоты, выявленные у детей с СРК причиной или следствием ФГИР [14].

Диагностические критерии СРК должны включать все нижеперечисленное:

- Абдоминальная боль по крайней мере 4 дня в месяц, связанная с одним или более из следующих критериев:

- дефекация,

- изменение частоты стула,

- изменение формы стула.

- У детей с запором боль не устраняется после купирования запора (дети, у которых боль устраняется после купирования запора, имеют функциональный запор, а не СРК).

- После соответствующей оценки симптомы не могут быть полностью объяснены другим состоянием.

Соответствие критериям должно наблюдаться в течение не менее 2 последних месяцев перед диагностикой.

СРК можно разделять на подтипы, базирующиеся на преобладании определенной формы кала у пациента, согласно Бристольской шкале формы кала (БШФК):[15]

СРК с преобладанием запора (РPК-3): более $25 \%$ дефекаций с формой стула 1 или 2 типа по БШФК типа и менее $25 \%$ дефекаций с формой стула 6 или 7 типа согласно БШФК. Альтернативный вариант для диагностики СРК-3 в клинической практике: пациент сообщает, что у него ненормальное опорожнение кишечника обычно в виде запора (напоминает типы 1 или 2 на картинке БШФК).

СРК с преобладанием диареи (СРК-Д): более $25 \%$ дефекаций с формой стула 6 или 7 типа согласно БШФК и менее 25\% дефекаций с формой стула типа 1 или 2 по БШФК. Альтернативный вариант: пациент сообщает, что у него ненормальное опорожнение кишечника обычно в виде диареи (напоминает типы 6 или 7 на картинке БШФК).

СРК смешанного вида (РPК-М): более 25\% дефекаций с формой стула типа 1 или 2 согласно БШФК и более $25 \%$ дефекаций с формой стула типа 6 или 7 по БШФК. Альтернативный вариант: пациент сообщает, что у него ненормальное опорожнение кишечника обычно бывает в виде и запора, и диареи (более $25 \%$ дефекаций - запор и более $25 \%$ дефекаций - диарея при использовании рисунка БШФК).

СРК неклассифицированный (СРК-Н): если у пациента, который имеет диагностические критерии СРК, нет возможности наверняка отнести расстройство к одному из трех типов СРК, то подтип следует отнести к неклассифицированному.

Учитывая, что СРК не имеет специфических симптомов, клиническая картина может маски- ровать другие заболевания кишечника. Тщательный сбор жалоб, данных анамнеза и клинический осмотр помогают провести дифференциальную диагностику СРК-3 с функциональным запором. В случае СРК-Д необходимо, в первую очередь, исключить кишечные инфекции, целиакию, синдром мальабсорбции, воспалительные заболевания кишечника. В то же время авторы консенсуса отмечают, что у большей части пациентов, имеющих признаки СРК, отсутствуют симптомы тревоги, и необходимость проведения диагностических исследований минимальная. В Римских критериях IV клиницистам рекомендуется выставлять диагноз СРК на основании клинической симптоматики и ограничивать арсенал диагностических мероприятий [16-18].

При опросе пациента обращают его внимание на обязательное наличие абдоминального болевого синдрома - отсутствие боли исключает СРК. Боль может локализоваться в любых отделах живота, но чаще - в нижних. Расстройства кишечника (запор, диарея) должны быть по времени возникновения ассоциированы с абдоминальным болевым синдромом. Пациента необходимо расспросить о характере питания, уделяя особое внимание употреблению злаков, кофеина, фруктов, овощей, соков, сладких напитков, использованию жевательной резинки, которые могут маскировать или усиливать симптомы СРК.

Лабораторное исследование пациентов с СРК включает общий анализ крови, С-реактивный белок, фекальный кальпротектин (используется как неинвазивный скрининговый тест для выявления воспалительных заболеваний кишечника). Если воспалительные маркеры слабо повышены, а вероятность воспалительных заболеваний кишечника мала, эксперты рекомендуют повторить лабораторные тесты до выполнения колоноскопии (если нет других показаний к ее проведению). Авторы обращают внимание на дифференцированный выбор тестов. Так, не рекомендовано определение провоспалительных маркеров у пациентов с запором; исследование уровня тиреоидных гормонов рутинно не показано всем пациентам (только при клинической необходимости); серологические тесты для диагностики целиакии (антитела к тканевой трансглутаминазе (IgA)) выполняют у пациентов с СРК-Д и СРК-М при отсутствии эффекта от эмпирического лечения; верхнюю эндоскопию с биопсией - при положительных серологических тестах и подозрении на целиакию; анализы кала (бактериологическое исследование, определение простейших, гельминтов) изучаются в странах с широкой распространенностью инфекци- 
онной диареи. Дыхательные тесты для уточнения мальабсорбции углеводов показаны у части пациентов с СРК-Д [19].

\section{Абдоминальная мигрень}

В согласительных документах указывается на частоту абдоминальной мигрени от $1 \%$ до $23 \%$ в детской популяции [20-22].

Дети с абдоминальной мигренью отмечают в качестве провоцирующих факторов: стресс, усталость или поездки, наличие схожих клинических симптомов (анорексия, тошнота и рвота), а в качестве факторов, вызывающих улучшение состояния: отдых и сон. В зрелом возрасте абдоминальная мигрень может трансформироваться в мигрень [23].

Диагностические критерии абдоминальной мигрени включают все приведенные ниже критерии и должны отмечаться по крайней мере дважды:

- Пароксизмальные эпизоды интенсивной острой периумбиликальной по средней линии или диффузной абдоминальной боли, которая длится 1 час и более (должен быть главным и наиболее тревожным симптомом).

- Эпизоды разделены периодами от недель до месяцев.

- Боль делает пациента нетрудоспособным и мешает нормальной деятельности.

- Стереотипная картина и симптомы у отдельных пациентов.

- Боль ассоциируется с двумя или более из следующих состояний:

- анорексия,

- тошнота,

- рвота,

- головная боль,

- светобоязнь,

- бледность.

- После соответствующей оценки симптомы не могут быть полностью объяснены другим состоянием.

Соответствие критериям должно наблюдаться в течение не менее 6 последних месяцев перед диагностикой [24].

Сочетание неспецифических продромальных симптомов, таких как изменение поведения или настроения, светобоязнь и вазомоторные реакции, похожие на те, что испытывают дети с мигренью, с улучшением состояния после приема амитриптилина подтверждает диагноз абдоминальной мигрени [25].

Необходимо исключить заболевания, связанные с органической патологией, основным симптомом которых является абдоминальный болевой синдром: урологические обструктивные процессы, обструкции пищеварительного тракта, заболевания желчевыводящих путей под- желудочной железы, а также психические расстройства [26].

\section{Функциональная неспецифическая абдоми-} нальная боль

Данное функциональное расстройство в Римские критерии IV включено впервые. Термин ФНАБ заменил существующую в Римских критериях III функциональную абдоминальную боль. В среднем от 35\% до 38\% детей начальной школы жалуются на боль в животе еженедельно. Лишь у трети из них симптомы соответствуют критериям для диагностики ФД, СРК или абдоминальной мигрени [12,20].

Диагностические критерии ФНАБ должны наблюдаться по крайней мере 4 раза в месяц и включают в себя все критерии из перечисленных ниже:

- Эпизодическая или продолжительная боль в животе, которая не связана исключительно с физиологическими событиями (например, прием пищи, менструация).

- Критерии недостаточны для СРК, ФД или абдоминальной мигрени.

- После соответствующей оценки абдоминальная боль не может быть полностью объяснена другим состоянием.

Соответствие критериям должно наблюдаться в течение не менее 2 последних месяцев перед диагностикой [21].

Дети с ФНАБ часто сообщают о неспецифических и внекишечных соматических симптомах, которые не обязательно требуют лабораторного или инструментального обследования. Особое внимание следует уделить наличию у пациента вегетососудистой дисфункции. В некоторых случаях для успокоения родителей выполняется ограниченное диагностическое обследование.

Лечение ФАБР является комплексным и должно начинаться с устранения провоцирующего фактора, коррекции режима дня и диетотерапии с исключением продуктов, вызывающих манифестацию симптомов. В плане медикаментозного лечения лишь немногие препараты доказали свою эффективность в проведенных рандомизированных клинических исследованиях. Особое внимание на сегодняшний день уделяется использованию определенных штаммов пробиотиков, а также координаторам моторики пищеварительного тракта, спазмолитикам (большинство из современных препаратов данной группы имеют возрастные ограничения для применения в педиатрической практике). Применение седативных препаратов, в т.ч. антидепрессантов, использование психотерапевтических методов рекомендовано к использованию лишь после консультации соответствующего специалиста [27-30]. 
Таким образом, ФАБР является достаточно распространенной патологией среди пациентов детского возраста. Изучение диагностических критериев данной группы заболеваний позволит своевременно поставить правильный ди- агноз без использования большого количества лабораторных и инструментальных исследований, своевременно назначить правильное лечение и повысить ее эффективность.

\section{А.В. Налетов ${ }^{1}$, Д.А Карпенко ${ }^{2}$, Л.Ф. Чалая ${ }^{1}$ \\ ${ }^{2}$ КУ «Центр первичной медико-санитарной помощи № 7 г. Донецка», Донецк \\ ДИФФЕРЕНЦИАЛЬНАЯ ДИАГНОСТИКА ФУНКЦИОНАЛЬНЫХ АБДОМИНАЛЬНЫХ БОЛЕВЫХ РАССТРОЙСТВ У ДЕТЕЙ В СВЕТЕ РИМСКОГО КОНСЕНСУСА IV}

${ }^{1}$ ГОО ВПО «Донецикий национальный медицинский университет имени М. Горького», Донецк

Функциональные гастроинтестинальные расстройства занимают одно из ведущих мест в структуре патологии органов пищеварения у детей. Одной из наиболее частых причин обращения за медицинской помощью пациентов различного возраста являются жалобы, связанные с наличием абдоминальной боли. Основополагающими рекомендациями по диагностике и лечению функциональных гастроинтестинальных расстройств у пациентов разного возраста являются Римские критерии IV. Функциональные абдоминальные болевые расстройства в педиатрической практике включают в себя «функциональ- ную диспепсию», «синдром раздраженного кишечника», «абдоминальную мигрень», «функциональную неспецифическую абдоминальную боль». В статье представлены основные диагностические критерии функциональных абдоминальных болевых расстройств в детском возрасте. Рассмотрены некоторые патогенетические механизмы данных заболеваний. Особое внимание уделено показаниям для углубленного лабораторного и инструментального обследования детей с функциональными гастроинтестинальными расстройствами.

Ключевые слова: функциональные абдоминаль-

ные болевые расстройства, дети, Римский консенсус IV. A.V. Nalyotov ${ }^{1}$, D.A. Karpenko ${ }^{2}$, L.F. Phalaya ${ }^{1}$

${ }^{1}$ SEI HPE «M. Gorky Donetsk National Medical University», Donetsk Municipal Institution «Donetsk primary medical care center № 7», Donetsk

\section{DIFFERENTIAL DIAGNOSIS OF FUNCTIONAL ABDOMINAL PAIN DISORDERS IN CHILDREN ACCORDING TO ROME IV}

Functional gastrointestinal disorders occupy one of the leading places in the structure of the pathology of the digestive system in children. One of the most frequent reasons for seeking medical care for patients of different ages are complaints related to the presence of abdominal pain. Rome IV is basic recommendations for the diagnosis and treatment of functional gastrointestinal disorders in patients of different ages. Functional abdominal pain disorders in pediatric practice include "functional dyspepsia”, "irritable bowel syndrome”, "abdominal mi-

\section{ЛИТЕРАТУРА}

1. Drossman D.A., Hasler W.L. Rome IV - Functional GI disorders: disorders of Gut-Brain interaction. Gastroenterology, 2016; 150(6): 1262-79.

2. Печкуров Д.В. Возрастные особенности функциональных расстройств желудочно-кишечного тракта, проявляющихся абдоминальными болями, с позиций биопсихосоциальной модели. Вопросы детской диетологии. 2015; 13 (2): 11-16.

3. Налетов А.В., Вьюниченко Ю.С., Коктышев И.В. Оцен- graine”, “functional nonspecific abdominal pain”. The article presents the main diagnostic criteria of functional abdominal pain disorders in childhood. Some pathogenetic mechanisms of these diseases are considered. Special attention is paid to the indications for in-depth laboratory and instrumental examination of children with functional gastrointestinal disorders.

Key words: functional abdominal pain disorders, children, Rome IV.

\section{REFERENCES}

1. Drossman D.A., Hasler W.L. Rome IV - Functional GI disorders: disorders of Gut-Brain interaction. Gastroenterology, 2016; 150(6): 1262-79.

2. Pechkurov D.V. Vozrastnye osobennosti funkcional'nyh rasstrojstv zheludochno-kishechnogo trakta, projavljajushhihsja abdominal'nymi boljami, s pozicij biopsihosocial'noj modeli. Voprosy detskoj dietologii. 2015; 13 (2): 11-16. (in Russian).

3. Naletov A.V., V'junichenko Ju.S., Koktyshev I.V. Ocen- 
ка качества жизни детей с синдромом раздраженного кишечника, проживающих в условиях военного конфликта в Донбассе. Университетская клиника. 2018; 2 (27): 12-16.

4. Трухан Д.И. Функциональные гастроинтестинальные расстройства: перспективы использования лечебной минеральной воды. Медицинский совет. 2017; 5: 7076.

5. Van Tilburg M.A.L., Walker L., Palsson O., et al. Prevalence of child/adolescent functional gastrointestinal disorders in a national U.S. community sample. Gastroenterology 2014; 144 (Suppl 1): S143-S144.

6. Hyams J.S., Burke G., Davis P.M., et al. Abdominal pain and irritable bowel syndrome in adolescents: a communitybased study. J. Pediatr. 1996; 129: 220-226.

7. Saps M., Pensabene L., Turco R., et al. Rotavirus gastroenteritis: precursor of functional gastrointestinal disorders? J. Pediatr. Gastroenterol. Nutr. 2009; 49: 580-583.

8. Saps M., Pensabene L., Di Martino L., et al. Post-infectious functional gastrointestinal disorders in children. J. Pediatr. 2008; 152: 812-816.

9. Schurman J.V., Singh M., Singh V., et al. Symptoms and subtypes in pediatric functional dyspepsia: relation to mucosal inflammation and psychological functioning. J Pediatr. Gastroenterol. Nutr. 2010; 51:298-303.

10. Guariso G., Meneghel A., Dalla Pozza L.V., et al. Indications to upper gastrointestinal endoscopy in children with dyspepsia. J. Pediatr. Gastroenterol. Nutr. 2010; 50: 493499

11. Saps M., Nichols-Vinueza D.X., Rosen J.M., et al. Prevalence of functional gastrointestinal disorders in Colombian school children. J. Pediatr. 2014; 164: 542-545.

12. Devanarayana N.M., Mettananda S., Liyanarachchi C., et al. Abdominal pain-predominant functional gastrointestinal diseases in children and adolescents: prevalence, symptomatology, and association with emotional stress. J. Pediatr. Gastroenterol. Nutr. 2011; 53: 659-665.

13. Iovino P., Tremolaterra F., Boccia G., et al. Irritable bowel syndrome in childhood: visceral hypersensitivity and psychosocial aspects. Neurogastroenterol. Motil. 2009; 21. 940-974.

14. Henderson P., Casey A., Lawrence S.J., et al. The diagnostic accuracy of fecal calprotectin during the investigation of suspected pediatric inflammatory bowel disease. Am. J. Gastroenterol. 2012; 107: 941-949.

15. Rajindrajith S., Devanarayana N.M. Subtypes and symptomatology of irritable bowel syndrome in children and adolescents: a school-based survey using Rome III criteria. J. Neurogastroenterol. Motil. 2012; 18: 298-304.

16. Horvath A., Dziechciarz P., Szajewska H. Meta-analysis: Lactobacillus rhamnosus GG for abdominal pain-related functional gastrointestinal disorders in childhood. Aliment. Pharmacol. Ther. 2011; 33: 1302-1310.

17. Guandalini S., Magazzu G., Chiaro A., et al. VSL\#3 improves symptoms in children with irritable bowel syndrome: a multicenter, randomized, placebo-controlled, double-blind, crossover study. J. Pediatr. Gastroenterol. Nutr. 2010; 51: 24-130.

18. Kline R.M., Kline J.J., Di Palma J., et al. Enteric-coated, $\mathrm{pH}$-dependent peppermint oil capsules for the treatment of irritable bowel syndrome in children. J. Pediatr. 2001; 138: 125-128.

19. Chumpitazi B.P., Hollister E.B., Oezguen N., et al. Gut microbiota influences low fermentable substrate diet efficacy in children with irritable bowel syndrome. Gut Microbes 2014; 5: 165-175.

20. Saps M., Adams P., Bonilla S., et al. Parental report of abdominal pain and abdominal pain-related functional gastrointestinal disorders from a community survey. J. Pediatr Gastroenterol. Nutr. 2012; 55: 707-710.

21. Devanarayana N.M., Adhikari C., Pannala W., et al. Prevalence of functional gastrointestinal diseases in a cohort of Sri Lankan adolescents: comparison between Rome II and Rome III criteria. J. Trop. Pediatr. 2011; 57: 34-39

22. Abu-Arafeh I., Russell G. Prevalence and clinical features of abdominal migraine compared with those of migraine ka kachestva zhizni detej s sindromom razdrazhennogo kishechnika, prozhivajushhih v uslovijah voennogo konflikta v Donbasse. Universitetskaja klinika. 2018; 2(27): 1216. (in Russian).

4. Truhan D.I. Funkcional'nye gastrointestinal'nye rasstrojstva: perspektivy ispol'zovanija lechebnoj mineral'noj vody. Medicinskij sovet. 2017; 5: 70-76. (in Russian).

5. Van Tilburg M.A.L., Walker L., Palsson O., et al. Prevalence of child/adolescent functional gastrointestinal disorders in a national U.S. community sample. Gastroenterology 2014;144 (Suppl 1): S143-S144.

6. Hyams J.S., Burke G., Davis P.M., et al. Abdominal pain and irritable bowel syndrome in adolescents: a communitybased study. J. Pediatr. 1996; 129: 220-226.

7. Saps M., Pensabene L., Turco R., et al. Rotavirus gastroenteritis: precursor of functional gastrointestinal disorders? J. Pediatr. Gastroenterol. Nutr. 2009; 49: 580-583.

8. Saps M., Pensabene L., Di Martino L., et al. Post-infectious functional gastrointestinal disorders in children. J. Pediatr. 2008; 152: 812-816.

9. Schurman J.V., Singh M., Singh V., et al. Symptoms and subtypes in pediatric functional dyspepsia: relation to mucosal inflammation and psychological functioning. J Pediatr. Gastroenterol. Nutr. 2010; 51:298-303.

10. Guariso G., Meneghel A., Dalla Pozza L.V., et al. Indications to upper gastrointestinal endoscopy in children with dyspepsia. J. Pediatr. Gastroenterol. Nutr. 2010; 50: 493499.

11. Saps M., Nichols-Vinueza D.X., Rosen J.M., et al. Prevalence of functional gastrointestinal disorders in Colombian school children. J. Pediatr. 2014; 164: 542-545.

12. Devanarayana N.M., Mettananda S., Liyanarachchi C., et al. Abdominal pain-predominant functional gastrointestinal diseases in children and adolescents: prevalence, symptomatology, and association with emotional stress. J. Pediatr. Gastroenterol. Nutr. 2011; 53: 659-665.

13. Iovino P., Tremolaterra F., Boccia G., et al. Irritable bowel syndrome in childhood: visceral hypersensitivity and psychosocial aspects. Neurogastroenterol. Motil. 2009; 21. 940-974.

14. Henderson P., Casey A., Lawrence S.J., et al. The diagnostic accuracy of fecal calprotectin during the investigation of suspected pediatric inflammatory bowel disease. Am. J. Gastroenterol. 2012; 107: 941-949.

15. Rajindrajith S., Devanarayana N.M. Subtypes and symptomatology of irritable bowel syndrome in children and adolescents: a school-based survey using Rome III criteria. J. Neurogastroenterol. Motil. 2012; 18: 298-304.

16. Horvath A., Dziechciarz P., Szajewska H. Meta-analysis: Lactobacillus rhamnosus GG for abdominal pain-related functional gastrointestinal disorders in childhood. Aliment. Pharmacol. Ther. 2011; 33: 1302-1310.

17. Guandalini S., Magazzu G., Chiaro A., et al. VSL\#3 improves symptoms in children with irritable bowel syndrome: a multicenter, randomized, placebo-controlled, double-blind, crossover study. J. Pediatr. Gastroenterol. Nutr. 2010; 51: 24-130.

18. Kline R.M., Kline J.J., Di Palma J., et al. Enteric-coated, $\mathrm{pH}$-dependent peppermint oil capsules for the treatment of irritable bowel syndrome in children. J. Pediatr. 2001; 138: $125-128$.

19. Chumpitazi B.P., Hollister E.B., Oezguen N., et al. Gut microbiota influences low fermentable substrate diet efficacy in children with irritable bowel syndrome. Gut Microbes 2014; 5: 165-175.

20. Saps M., Adams P., Bonilla S., et al. Parental report of abdominal pain and abdominal pain-related functional gastrointestinal disorders from a community survey. J. Pediatr Gastroenterol. Nutr. 2012; 55: 707-710.

21. Devanarayana N.M., Adhikari C., Pannala W., et al. Prevalence of functional gastrointestinal diseases in a cohort of Sri Lankan adolescents: comparison between Rome II and Rome III criteria. J. Trop. Pediatr. 2011; 57: 34-39

22. Abu-Arafeh I., Russell G. Prevalence and clinical features of abdominal migraine compared with those of migraine headache. Arch. Dis. Child. 1995; 72: 413-417. 
headache. Arch. Dis. Child. 1995; 72: 413-417.

23. Rasquin-Weber A., Hyman P.E., Cucchiara S., et al. Childhood functional gastrointestinal disorders. Gut. 1999; 45 (Suppl. 2): II60-II68.

24. Catto-Smith A.G., Ranuh R. Abdominal migraine and cyclical vomiting. Semin. Pediatr. Surg. 2003; 12: 254-258.

25. Symon D.N., Russell G. Abdominal migraine: a childhood syndrome defined. Cephalalgia. 1986; 6: 223-228.

26. Pourmoghaddas Z., Saneian H., Roohafza H., et al. Mebeverine for pediatric functional abdominal pain: a randomized, placebo-controlled trial. Biomed. Res. Int. 2014; 2014: 191026

27. Saps M., Youssef N., Miranda A., et al. Multicenter, randomized, placebo-controlled trial of amitriptyline in children with functional gastrointestinal disorders. Gastroenterology. 2009; 137: 1261-1269.

28. Bahar R.J., Collins B.S., Steinmetz B., et al. Double-blind placebo-controlled trial of amitriptyline for the treatment of irritable bowel syndrome in adolescents. J. Pediatr. 2008; 152: 685-689.

29. Roohafza H., Pourmoghaddas Z., Saneian H., et al. Citalopram for pediatric functional abdominal pain: a randomized, placebo-controlled trial. Neurogastroenterol. Motil. 2014; $26: 1642-1650$.
23. Rasquin-Weber A., Hyman P.E., Cucchiara S., et al. Childhood functional gastrointestinal disorders. Gut. 1999; 45 (Suppl. 2): II60-II68.

24. Catto-Smith A.G., Ranuh R. Abdominal migraine and cyclical vomiting. Semin. Pediatr. Surg. 2003; 12: 254-258.

25. Symon D.N., Russell G. Abdominal migraine: a childhood syndrome defined. Cephalalgia. 1986; 6: 223-228.

26. Pourmoghaddas Z., Saneian H., Roohafza H., et al. Mebeverine for pediatric functional abdominal pain: a randomized, placebo-controlled trial. Biomed. Res. Int. 2014; 2014: 191026.

27. Saps M., Youssef N., Miranda A., et al. Multicenter, randomized, placebo-controlled trial of amitriptyline in children with functional gastrointestinal disorders. Gastroenterology. 2009; 137: 1261-1269.

28. Bahar R.J., Collins B.S., Steinmetz B., et al. Double-blind placebo-controlled trial of amitriptyline for the treatment of irritable bowel syndrome in adolescents. J. Pediatr. 2008; 152: 685-689.

29. Roohafza H., Pourmoghaddas Z., Saneian H., et al. Citalopram for pediatric functional abdominal pain: a randomized, placebo-controlled trial. Neurogastroenterol. Motil. 2014; 26: 1642-1650. 